\title{
The Approach to a Major Incident
}

\author{
Maj RJ Russell \\ MB BS, MRCP(UK), Dip IMC RCSEd, RAMC \\ Specialist Registrar in Accident and Emergency Medicine
}

MDHU Frimley Park Hospital, Portsmouth Road, Frimley, Surrey GU16 5UJ

SUMMARY: Any major incident will test the organisational and medical skills of those involved. The best response can only be achieved with good planning and preparation, and the adoption of a structured approach to the scene. A clear chain of command should exist within all emergency services and must be followed. The Ambulance and Medical Incident officers take overall responsibility for the health service response. Management priorities - Command and Control, Safety, Communications, Assessment - must take precedence over support priorities - Triage, Treatment, Transport.

\section{Introduction}

A major incident for the health service professional is a huge challenge for the medical staff involved in the response. Their medical skills are likely to be severely tested on individual patients, with the added complications of a confused environment, limited resources and multiple casualties. This scenario is closer to that expected on the battlefield by the military Medical Officer than that experienced by civilian doctors.

An optimum response can only be achieved by using a structured method. This will ensure that management as well as medical priorities are met. A degree of order can then be established out of the inevitable chaos.

This article is based on the Major Incident Medical Management and Support (MIMMS) approach, which is applicable to any major incident. It will deal only with the prehospital response to a major incident.

\section{What is a Major Incident?}

A major incident is any event in which the live casualties by their number, type or location require extraordinary resources. A major incident for one emergency service may not be a major incident for the others. In the initial confusion, however, it is likely that all the emergency services will declare a major incident before the exact magnitude and nature of the incident is clear. The possibility of over-reaction is necessary as it is easier to stand services down once it is clear that they are no longer required, than it is to mobilise them late.

Major incidents may be categorised as natural or man-made, simple or compound and compensated or uncompensated. A compound incident involves damage to the surrounding infrastructure (roads, communications etc). An uncompensated incident will produce more casualties than can be dealt with even after mobilisation of extraordinary resources.

Major incidents may be the result of accidents, negligence or deliberate acts. The incident site must therefore be treated as a scene of crime, and rescue personnel must be aware that there will almost certainly be an inquiry into the incident and the response to it. All notes, photographs and tapes from dictaphones (used as a method of making notes) are legal documents and must be treated as such.

The major aims of the health services at the scene are as follows

1. Save life

2. Prevent further harm to the injured

3. Relieve suffering

\section{Planning and Preparation}

Improving the capacity for optimal performance at a major incident begins well before it happens, on both personal and organisational levels. Medical staff arriving at the scene of a major incident without adequate personal protective equipment will not get the chance to give any sort of performance at all. This phase can be looked at as three separate areas - planning, equipment and training.

Planning - The exact nature of a major incident can never be predicted in full. Planners must therefore adopt an 'all hazards approach'. However, in any given area high risk points can be identified (e.g chemical plants, sports stadia etc) and specific plans should exist to cover them. Medical Officers must identify these points and make themselves familiar with the plans. No plan can cover all eventualities and plans should be flexible as a rigid plan will be thrown into confusion when disrupted by events.

If no general or specific plans exist then attention must be drawn to this and plans made. Assistance with all aspects of planning is available through the local Emergency Planning Officer, and guidance is given in NHS documents (see further reading).

Equipment - Any medical staff who may deploy to a major incident must be familiar with both personal protective and medical equipment.

Personal protective equipment (PPE) - This must provide protection against all possible hazards at the scene as well as being comfortable, durable and functional. Medical staff arriving inappropriately dressed will not be allowed on to the scene. Box 1 shows the minimum requirements. Colouring and pattern should conform to local standards. Rescuers should also have other personal equipment prepared to meet their own requirements - ID, notebook, aide memoire, torch, polaroid camera, whistle and comfort pack for example.

\section{Box 1 - Minimum clothing requirements}

Helmet/Hard hat with chin strap -
side torch advisable
Goggles/Visor
Ear defenders
Suit - fire-retardant
Jacket - high visibility
Gloves - heavy duty and latex
Boots - oil and chemical resistant

Medical Equipment - Each ambulance/medical practitioner will bring a limited amount of equipment to the scene. All medical staff should be familiar with their own equipment and the way it is packed. Packaging should be easily portable. Equipment must be limited to that needed for appropriate treatment at the scene. Resupply is the responsibility of the ambulance service. This will be organised via the chain of command and all requests must go through the chain to avoid duplication and confusion. Ambulance services have designated resupply vehicles, and an equipment 
depot will usually be set up at the scene to optimise the use of scarce resources. There is no national standard for emergency medical equipment so medical officers must ensure that their kit is compatible with that of the local ambulance service.

Training - This should be considered as two separate procedures - education and exercise.

Education consists of teaching sessions, reading and courses so that knowledge and skills are acquired.

Exercise is practising the knowledge and skills so that the first time they are tested is not for real. The same holds true for emergency plans. Exercises can vary in scale from simple paperwork practises to full dummy incidents complete with mock casualties. Emergency equipment packs should be regularly unpacked and checked. This familiarises staff with the contents and layout and avoids the embarrassment of arriving with out-ofdate drugs.

\section{Initial actions at the scene}

The timing of arrival will determine the initial actions of the doctor. If the response is already under way, the doctor should report to the medical Incident Officer (MIO) to book in and receive briefing and instructions. However, if arriving as the first doctor on the scene then the role of MIO must be taken on. This will be held until an MIO designated by the ambulance service arrives to take over. Each ambulance service should have a pool of trained and equipped doctors available to act as MIO.

The phrase "Safely Control then ACT" summarises the actions needed immediately after arrival very early in the incident (Box 2). This approach can be applied to any incident large or small.

\section{Box 2 - Initial actions on scene}

Safely - Safety is paramount

Control

A - Assess

C - Communicate

$\mathrm{T}$ - Treat

Safety is the overriding concern. Rescuers must not become casualties themselves. Don protective equipment. Ask the questions 1. am I safe? 2. is the scene safe? 3. are the casualties safe?

Establish control over the situation - the police will take over this role on arrival.

Assess the whole incident quickly with particular regard to safety and gathering information that ambulance control will require.

Communicate with Ambulance Control so that help will start moving in your direction. Declare a major incident so that plans can be activated early. Delay in declaring a major incident, often for fear of embarrassment if wrong, will lead to a slow and patchy response. This will lead to increased mortality, morbidity and suffering amongst the casualties. If there is doubt a "standby" message can be passed to alert control to inform key staff without leading to full mobilisation. The mnemonic "ETHANE" contains the information which must be passed to ambulance control (Box 3).

\section{Box 3 - Initial communication information}

(M) - (Major Incident declared - if not already done)

E - Exact Location (grid reference if possible)

$\mathrm{T}$ - Type of incident (e.g. rail, airplane)

$\mathrm{H}$ - Hazards

A - Approaches to be used

$\mathrm{N}$ - Number of casualties (approximate numbers, type and severity)

E - Emergency services present at scene and required
Treatment should be instigated but the lone doctor, acting as MIO, must not become involved with the treatment of individual casualties. If this happens the wider picture will be lost and more important priorities neglected.

\section{Priorities at the scene}

The further management of a major incident should be considered under the priorities shown in Box 4. These priorities can be remembered as "CS CAT with 3 tails".

\section{Box 4 - Priorities at the scene}

Command and control
Safety
Communications
Assessment
Triage
Treatment
Transport

Command and Control - Each emergency service will appoint an Incident Officer (IO: Police-PIO etc). Each IO will take command of that service's resources at scene and assume responsibility for all aspects of its performance. Each IO should wear an appropriately coloured tabard clearly labelling their role. It is vital that discipline is maintained and each service's chain of command observed. The police are in overall control of the incident. Different levels of command and control exist.

The operational (Bronze) level is the area where the rescue is taking place. The IO's may appoint Forward Incident Officers (FPIO etc.) to oversee this area and provide them with better information from close in. In some scenarios (e.g. rail crash with several carriages involved) the IO's may sectorize the incident creating more than one bronze area, each with a set of Forward Officers. If a hazard still exists in this area the FFIO may have control at this level.

The tactical (Silver) level encompasses the whole incident and the immediate response. This is the area commanded by the IO's. It will be cordoned off by the police.

The strategic (Gold) level consists of the Chief officers of each emergency service and the Local Authority. They will meet at a suitable location to co-ordinate the larger response over a county or even country-wide area.

Police - The police have overall control of the incident, and their aims and responsibilities are shown in Box 5. By establishing control and order the police facilitate the roles of the other services. The first police officer on scene will act as PIO, and this role will be relinquished to increasingly senior officers as they arrive.

\section{Box 5 - Aims and Responsibilities of the Police}

Control of the incident and surrounding area

- prevent escalation

- traffic control

- maintain public order

- protect environment

- protect property

Save life

Liaison with other services

Evacuation if continuing threat

Collection/Dissemination of information on all involved in incident - Casualty Bureau

- Police documentation teams at receiving hospitals

- Survivor reception centre for uninjured

- Relatives reception centre 
Supervision of Voluntary Services and other volunteers

Identification of the dead - act as Coroner's officers

Investigation of the scene - scene of crime unless natural disaster

Liaison with Media

The Fire Service - Their main role is the control and/or elimination of hazards. Other aims and responsibilities are shown in Box 6. When the initial call is made, the fire service control room will dispatch the "pre-determined attendance". This ensures that the fire service response matches the perceived threat. The senior officer with this response will be the FIO until replaced by a senior.

\section{Box 6 - Aims and Responsibilities of the Fire Service}

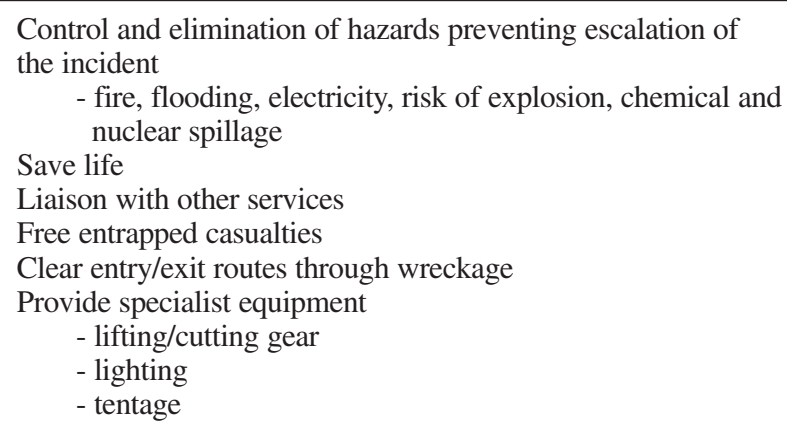

The Ambulance Service - Assessment, treatment and transfer are the primary objectives of the ambulance service. On the arrival of the first ambulance at the scene one of the crew, preferably a paramedic, will become AIO and should quickly assess the situation. The vehicle will become the initial Forward Ambulance Control Unit (FACU) and the second member of the crew should stay with it to provide communications to Ambulance Control. As the FACU this ambulance should be the only one to leave its beacon on. Once Control have been given a preliminary report, the AIO should begin to select important areas such as the Casualty Clearing Station (CCS), make key appointments and allocate duties as other staff arrive. Ambulance service aims and responsibilities are shown in Box 7. A schematic representation of the scene with key ambulance and medical appointments is shown in Figure 1.

Ambulance Control will activate the Emergency Reserve Channel (ERC). This allows communications concerning the major incident to be kept separate from the continuing every-day operations. Control will also dispatch a senior manager to take over as AIO, an Emergency Control Vehicle with extra communication equipment, and an Emergency Supply Vehicle. An Equipment Officer will be appointed by the AIO to pool all equipment arriving at the scene and distribute it as required, as well as arranging for resupply.

\section{Box 7 - Ambulance service aims and responsibilities}

\section{Save life}

Prevention of further injury and suffering

Triage

Transport and documentation of movement of casualties

Provision of communications for health service staff at scene

Mobilisation of available medical resources

- Immediate Care Doctors

- Voluntary Ambulance Services

Determine receiving hospitals and capacities

Liaison with other services

Set up CCS plus ambulance parking and loading points

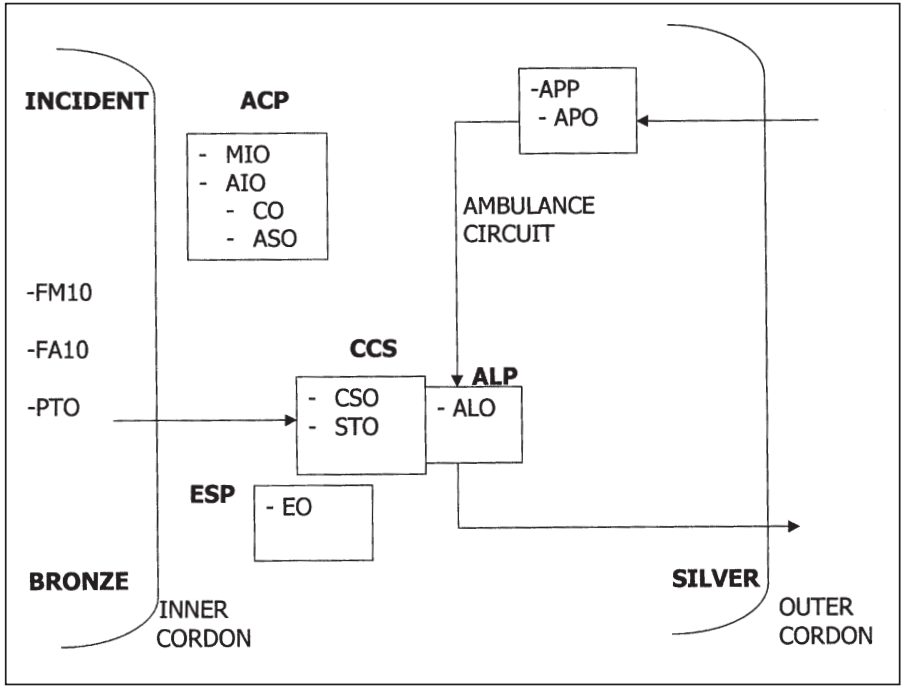

Fig 1. Layout of health services at scene. FM10/FA10Forward medical/Ambulance Incident officer. PTOPrimary Triage Officer. ACP-Ambulance Control Point. MIO/AI0- Medical/Ambulance Incident Officer. COCommunications Officer. ASO- Safety Officer. CCSCasualty Clearing Station. CCO- Casualty Clearing Officer. STO- Secondary Triage Officer. ESP- Equipment Supply Point. EO- Equipment Officer. APP/O- Ambulance Parking Point/Officer. ALP/O- Ambulance Loading Point/Officer

Medical Services - The MIO takes responsibility for the medical resources at the scene. All medical and nursing staff must report to the MIO on arrival for briefing and tasking. The MIO and the AIO must work very closely together to ensure maximum efficiency and minimum duplication as there is a large overlap in their roles. The duties of the MIO are summarised in Box 8 .

\section{Box 8 - The duties of the MIO}

Take responsibility for all medical resources at scene Appoint other medical or nursing staff to key roles Liaise closely with the AIO and other IO's

Communicate with senior staff at receiving hospitals

Assess the scene with regard to deployment of medical resources Assess the scene with regard to further mobilisation of medical resources

Liaise with AIO to obtain further/specialist medical resources

Liaise with AIO to decide which patients will go to which hospitals Establish and oversee triage and treatment

Mobile Medical Teams (MMT) may be requested from hospitals by the AIO and MIO. All hospitals with a 24 hour Accident and Emergency department are required to provide an MMT. It is important that the MMT's do not come from the hospitals closest to the incident, which will be the primary receiving hospitals. The ideal formation of an MMT is an experienced emergency physician as team leader accompanied by an anaesthetist and two experienced casualty nurses. MMT's can be tasked to various duties at the scene or the CCS, either as a complete team or individuals.

Extraction techniques and equipment have advanced to the point where amputation is rarely required at the scene. A Mobile Surgical Team (MST) should only be summoned by the AIO and MIO on a task-specific basis. A senior surgeon should replace the emergency physician in the team formation and an ODA or theatre nurse one of the casualty nurses, and the team should accompany the patient to a receiving hospital 
on completion of their task.

Other agencies - There are several other agencies, which can provide assistance when requested. These include the Coastguard, the military and the Voluntary Ambulance Services. The Salvation Army and the WRVS can be invaluable in manning the Survivor Reception Centre and rest areas for the rescue workers. The Local Authority will have the prime role in returning the community to long-term normality, but can also provide assistance to the rescue effort at the scene.

Safety - All rescue workers at the site must be keenly aware of personal safety, site safety and patient safety. In addition, a key appointment made by the AIO is that of Ambulance Safety Officer (ASO). This officer is responsible to the AIO for all aspects of health and safety for the health service workers on site. He should ensure that all staff have the correct PPE and are given adequate rest breaks. He should liaise with the other services, especially the fire service, with regard to hazard identification, evacuation, protective measures and decontamination.

Communications - The quality of the communications at and around the scene will determine the quality of the response. Poor communications have been identified as a failing at many of the inquiries into major incidents.

Immediately after a major incident is declared Ambulance Control should dispatch an Emergency Control Vehicle to the scene. This vehicle will become the Ambulance Control Point and has the necessary equipment for on and off-scene communications. The AIO should appoint a Communications Officer to co-ordinate and record all communications. Handheld radios are the main-stay of on-scene communications. Radio operation and voice procedure should have been practised as part of training and must be strictly adhered to. If the incident involves a known or suspected bomb then radios must not be used until permission to do so is given by the police in case a secondary device is triggered. Cellular telephones are increasing in popularity and avoid some of the problems of radios - lack of practise, voice procedure. Their use does, however, compromise the chain of command as there is no central control. The available cells will quickly become blocked, especially after the media arrive on the scene and start reporting. Access Overload Control (ACCOLC) can be instigated by the police allowing only registered and modified telephones to function.

Many other methods of communications from the simple (runner, megaphone) to the advanced (land line, fax, satellite link-up) may be used depending on the incident. Hand signals and whistles can be effective but many cause confusion unless use and meaning has been agreed in advance. Face-to-face communication between the IO's at regular liaison meetings is essential.

Assessment - The initial assessment included safety, the number and type of casualties and the emergency services present and required. Inevitably this information will be approximate and the AIO and MIO should be continually reassessing the scene. More accurate and up to date information can be used to request more supplies to MMT's and update the strategic planners and receiving hospitals.

Triage - Triage is the process by which casualties are sorted and assigned priorities for treatment and transfer, and is a concept with which all military doctors should be familiar. It is a dynamic process and should be repeated whenever a casualty is reassessed or arrives in a new location. Table 1 shows the triage priorities using both the 'P' (priority) and " $\mathrm{T}$ " (treatment) systems.

The decision to invoke the "expectant" category is a difficult one and should be made by the AIO and MIO together.
Table 1 Triage priorities

\begin{tabular}{|llll|}
\hline Type & "P" & "T" & Colour \\
\hline Immediate & 1 & 1 & Red \\
Urgent & 2 & 2 & Yellow \\
Delayed & 3 & 3 & Green \\
Expectant & & 4 & Blue \\
Dead & Dead & Dead & White \\
\hline
\end{tabular}

The AIO will appoint a Primary Triage Officer to carry out and supervise the triage at the scene. The MIO will appoint a Secondary Triage Officer to triage at the CCS. Once a priority has been assigned, the patient must be clearly labelled. There are many labelling systems available and doctors should familiarise themselves with the system in local use.

Treatment - The aim must be to identify and treat immediately life-threatening conditions and to stabilise the patient for transfer to hospital. Most treatment will therefore concentrate on the ABC's. It is essential that senior staff keep an overview and ensure effective triage rather than plunging into the treatment of one particular patient.

The AIO and MIO will select a site for the CCS and each appoint a Casualty Clearing Officer. The CCS must be safe and where possible available shelter should be used. The CCS should be easily accessible for teams bringing casualties to and ambulances evacuating from it. If no shelter is available then treatment should start as soon as equipment and casualties arrive, and not wait for tents to be erected.

The Casualty Clearing Officers should supervise all triage and treatment within the CCS, and ensure treatment and movements are recorded. Casualty and equipment states should be monitored and regularly fed back to the AIO and MIO. Transport requirements should be communicated to the Ambulance Loading Officer.

Transport - Efficient use of all transport resources is vital to the response. Transport at the scene to the CCS will usually require stretchers and man-power. Some patients may by-pass the CCS and go direct to the ambulance loading point. The AIO should appoint an Ambulance Loading Officer and an Ambulance Parking Officer. The Loading Officer should establish an ambulance circuit and ensure in liaison with the AIO, MIO and Casualty Clearing officers that patients are evacuated in the appropriate order using appropriate transport (e.g. buses can be used for the priority 3 patients). The Parking Officer should establish a parking point to which all ambulances are directed on arrival, $\log$ ambulances and their crews on and off site and, with the Loading Officer, ensure a regular flow of vehicles through the loading point.

It is important that patients are spread around the receiving hospitals, arriving in spaced "pulses". This will avoid flooding the nearest Casualty Department and compromising the care of the patients there. The P3 casualties may be sent to the more distant hospitals, and consideration should be given to sending certain casualties (e.g. burns or head injuries) direct to specialist centres.

\section{The Dead}

Death can only be pronounced by a doctor. This should be done in the presence of a police officer to act as the Coroner's representative. If, during triage, a paramedic or other rescue worker diagnoses death the casualty must be labelled as such. This will stop other rescuers repeatedly reassessing the body.

Unless it is the result of a natural disaster, the site must be treated as a scene of crime. Bodies must be left unmoved except 
where they are preventing access to live casualties or are in danger of destruction by fire or corrosive chemicals. If movement has to take place then it should be fully documented and marked and, where possible a photograph taken. The MIO will appoint a doctor to be Mortuary Officer. This doctor's duties are to declare death and, liaising with the police, set up a bodyholding area. This may not be required if there is a nearby temporary mortuary, identified during emergency planning, where the bodies can be examined by a forensic pathologist. Identification of the dead is police responsibility.

\section{The Aftermath}

Major incidents are beyond the experience of most rescue workers. A detailed debrief for all involved should be held within 48 hours of the incident finishing. This debrief should be on two levels. Firstly the operational aspects should be addressed. What went well? What could we have done differently? What lessons can be learned and incorporated into planning? Secondly, the psychological aspects can be acknowledged so that staff are aware of what normal reactions they may feel, and what arrangements have been made for counselling for those who wish it.

\section{The Media}

Any major incident will attract a vast amount of interest from the media, whose response will be almost as rapid as that of the emergency services. Properly managed, the media can be a useful method of disseminating information to the public about which areas to avoid and which telephone numbers to ring for casualty information. Badly managed, the media can obstruct the rescue effort as they attempt to gather information and footage for the next bulletin.

The police have responsibility for media liaison and will often provide a media liaison officer, who can give regular briefings. A rendezvous point should be arranged for all media personnel and other facilities arranged as circumstances allow. All staff need to be briefed on how to respond to requests from the media and the IO's should expect to have to give interviews at some point. The likely points that the media will want to cover are what has happened, why has it happened and who is to blame? Answers should be confined to facts and speculation avoided.

\section{Conclusion}

A major incident will stretch the management and clinical capabilities of all health service staff involved. A structured response will help provide optimum care to the injured and reduce loss of life and suffering.

\section{Further reading/training}

Major Incident Medical Management and Support - the practical approach

Advanced Life Support Group

BMJ Publishing 1995

MIMMS 3 day courses - contact RDMC or

Advanced Life Support Group
Second Floor
The Dock office
Trafford Road
Salfords Quays
Manchester
M5 2XB

Emergency planning in the NHS: health services arrangements for dealing with major incidents - accompanies $\mathrm{HC}(90) 25$ and HSG(93)24 HMSO 